\title{
PREMATURIDADE DE CRIANÇAS NASCIDAS NO CENTRO OBSTÉTRICO DO MUNICIPIO dE COARI - AMAZONAS, BRASIL
}

\section{Prematurity of children born at the obstetric center of the municipality of Coari - Amazonas, Brazil}

Ana Felisa Hurtado Guerrero', Silvaney Gonçalves Reis², Perpétua Souza da Costa Parente $^{3}$, Daiane Valéria Oliveira da Silva ${ }^{4}$, José Camilo Hurtado Guerrero ${ }^{5}$

\begin{abstract}
1 Gerontóloga. Sanitarista e ex-docente do curso de Bacharelado em Saúde Coletiva da Universidade do Estado do Amazonas, Escola de Ciências da Saúde, Manaus, Amazonas, Brasil. ORCID: https://orcid. org/0000-0002-2816-6235

2 Técnica de Enfermagem da Secretaria Estadual de Saúde no Município de Coari. Bacharel em Saúde Coletiva pela Universidade do Estado do Amazonas, Escola de Ciências da Saúde, Manaus, Amazonas, Brasil. ORCID: https://orcid.org/0000-0002-1537-1491

3 Técnica de Análises Clínicas da Secretaria Municipal de Saúde do Município de Coari. Bacharel em Saúde Coletiva pela Universidade do Estado do Amazonas, Escola de Ciências da Saúde, Manaus, Amazonas, Brasil. ORCID: https://orcid.org/0000-0003-2784-5399

4 Técnica em Enfermagem da Secretaria Municipal de Saúde do Município de Coari. Bacharel em Saúde Coletiva pela Universidade do Estado do Amazonas, Escola de Ciências da Saúde, Manaus, Amazonas, Brasil. ORCID: https://orcid.org/0000-0002-1607-6515

5 Biólogo - Entomólogo. Professor Visitante Nacional do Instituto de Biodiversidade e Floresta da Universidade Federal do Oeste do Pará (UFOPA), Santarém, Pará, Brasil. ORCID: https://orcid.org/00000001-9062-1860
\end{abstract}

CONTATO: José Camilo Hurtado Guerrero | Universidade Federal do Oeste do Pará | Instituto de Biodiversidade e Florestas (IBEF) - Campus Tapajós | Rua Vera Paz, s/n | Salé | Santarém - PA | CEP: 68040-470. | E-mail: jhocamhur@gmaill.com

COMO CITAR: Guerrero AFH, Reis SG, Parente PS, Silva VO, Guerrero JCH. Prematuridade de crianças nascidas no Centro Obstétrico do município de Coari-Amazonas, Brasil. R. Saúde Públ. 2018 Dez;1(2):23-34

(c) (1) COPYRIGHT Esta obra é disponibilizada nos termos da Licença Creative Commons - 4. 0 Internacional. É permitida a reprodução parcial ou total desta obra, desde que citada a fonte.

RESUMO OBJETIVO: Analisar a incidência de partos prematuros no Centro Obstétrico de Coari, no período de 2011 a 2015. MÉTODO: Estudo retrospectivo quantitativo sobre partos prematuros no Hospital Regional de Coari Odair Carlos Geraldo. RESULTADOS: Mostrou-se que foram mães adolescentes, solteiras, agricultoras com intercorrências como contrações uterinas e parto normal. Nos partos prematuros nasceram mais meninos com peso 
normal e entre 35 e 37 semanas. Em relação ao índice de Apgar, 10,6\% dos prematuros obtiveram pontuação menor ou igual a sete no primeiro minuto de vida, sendo que no quinto minuto esse percentual caiu para 7,8\%, indicando que a maioria teve uma rápida recuperação. CONCLUSÃO: A gravidez na adolescência vem crescendo no município e com isso aumentando também os partos prematuros, portanto os cuidados devem ser centrados na realização de um bom pré-natal para evitar complicações, uma vez que não contam com UTI neonatal.

PALAVRAS-CHAVE: Trabalho de Parto. Parto Prematuro. Recém-Nascido Prematuro.

ABSTRACT OBJECTIVE: To analyze the incidence of premature deliveries at the Obstetric Center of Coari in the period from 2011 to 2015. METHODS: This is a retrospective, quantitative study on premature deliveries at the Regional Hospital of Coari Odair Carlos Geraldo. RESULTS: Findings showed that most mothers were adolescents, single, farmers who had complications such as uterine contractions, and normal birth. Regarding premature newborns, there were more boys with normal weight, born between 35 and 37 weeks. Regarding the Apgar score, 10.6\% of premature infants scored seven or less in the first minute of life, and in the fifth minute this percentage fell to $7.8 \%$, indicating that most of them had a rapid recovery. CONCLUSION: Adolescent pregnancy has been increasing in the municipality and, with it, premature births. Thus, care should focus on good prenatal care to avoid complications, because they cannot rely on a neonatal Intensive Care Unit (ICU).

KEYWORDS: Labor of Delivery. Premature birth. Premature Newborn.

\section{INTRODUÇÃO}

$\mathbf{0}$ parto pré-termo ou prematuro é aquele que ocorre quando a gestação é interrompida depois da $20^{a}$ semana de gravidez e antes ou igual à $37^{\mathrm{a}}$ semana de gestação, um número que corresponderia ao tempo mínimo para o desenvolvimento do feto, sendo a prematuridade uma das principais causas de mortes neonatais ${ }^{1.2}$. Considera-se prematuridade grave o escore do Apgar entre 0 a 3, de 4 a 7 moderado e de 8 a 10 normal ${ }^{3}$. A prematuridade é classificada de acordo com sua evolução clínica, sendo ela eletiva, onde a complicação estão em decorrência de problemas que estão ocorrendo com a mãe como por exemplo: doenças hipertensivas, descolamento da placenta, dentre outras. Ou espontânea, na qual não se detecta nenhuma complicação da mãe $e^{4.5}$.

As causas mais comuns de parto prematuro estão relacionadas com a faixa etária, a desnutrição, o baixo peso durante a gestação, tratamento de infertilidade, complicações obstétricas, as infecções vulvovaginais, a extensão do cuidado no pré-natal, fatores comportamentais, o que torna essencial o acompanhamento das gestantes e de algumas doenças específicas durante este período de gestação para evitar um parto pré-termo ${ }^{6,7.89}$.

Geralmente o parto pré-termo tem maior ocorrência em adolescentes com idade inferior a 
dezenove anos e mulheres com gestações tardias? Nesses grupos etários, a grande atenção deve estar voltada aos riscos de morbimortalidade perinatais, especialmente, nas adolescentes que ainda estão em fase de crescimento e não apresentam certa maturidade fisiológica, o que as torna incapazes de suportar adequadamente o estresse característico da gestação ${ }^{10}$. Assim, os problemas que ocorrem durante o processo do ciclo gravídico puerperal podem ser fatores de risco à saúde tanto da mãe quanto do feto e podendo evoluir para a morte do neonato. ${ }^{5}$

Em muitos casos, além dos gastos financeiros, e dos cuidados dos profissionais de saúde e dos próprios familiares, o parto prematuro pode ocasionar problemas na qualidade de vida do neonato e também no desenvolvimento psicossocial da criança11. Embora a chance de sobrevida dos recém-nascidos prematuros tenha aumentado nos últimos anos, devido às tecnologias existentes, a prematuridade ainda vem sendo a principal causa de morbidade e mortalidade neonatal, sendo um dos grandes desafios para a obstetrícia. Por isso, apresenta-se como um dos maiores problemas encontrados em mulheres gestantes, tanto em países desenvolvidos como em desenvolvimento ${ }^{2.5}$.

O parto prematuro é considerado um grande problema para a saúde pública, pois, segundo a estimativa, nascem cerca de 15 milhões de bebês prematuros por ano no mundo, determinando que sua ocorrência seja de um parto prematuro em cada dez nascimentos ${ }^{12}$. Em nível mundial em 2010 aconteceram cerca de 15 milhões de partos prematuros, e cerca de um milhão desses recémnascidos morreram após o parto, ou por algum tipo de complicação ${ }^{13}$. Enquanto que no Brasil são registrados mais de 279 mil nascimentos de recémnascidos pré-termos totalizando cerca de 17,7\% dos partos realizados ${ }^{14}$, sendo que o nascimento prétermo é a maior causa de morte neonatal no pais, tendo um percentual de $70 \%$ dos casos $^{6,15}$.

A Região Norte é responsável por 9\% dos nascimentos totais e $6 \%$ são pré-termos de muito baixo peso. No entanto, em Manaus, capital do Amazonas, observou-se um aumento no percentual de prematuridade de 3,8\% para $7 \% 5$. Este estudo teve como objetivo analisar a incidência de partos prematuros no Centro Obstétrico de Coari, no período de 2011 a 2015.

\section{METODOLOGIA}

Realizou-se um estudo descritivo, retrospectivo e de abordagem quantitativa a partir dos prontuários de crianças prematuras nascidas de mães admitidas no Centro Obstétrico de assistência e cuidado de puérperas e neonatos do Hospital Regional de Coari Dr. Odair Carlos Geraldo (HRCOCG), residentes na região do Médio Solimões e que tiveram seus partos assistidos no período de 2011 a 2015. O Centro de referência é responsável também pelo atendimento de grávidas de municípios circunvizinhos, localizados no Médio Solimões, como são: Tefé, Alvarães, Uarini, Codajás, Anori, Anamã e Beruri.

A coleta de dados foi realizada no Setor de Estatística do Hospital, através dos prontuários e fichas de internação das puérperas, totalizando um universo de 957 prematuros. Na definição de crianças prematuras se utilizou como referência a tabela de escores de Apgar de Santos e Pasquini ${ }^{3}$. para os nascidos prematuros com menos de 37 semanas.

O Apgar é um método de avaliação sistemática do recém-nascido, realizado após o parto, que avalia as condições fisiológicas e a capacidade de resposta, e serve para identificar se a criança necessita de reanimação ou cuidados especiais ${ }^{3}$. O Apgar avalia cinco sintomas da saúde do recém-nascido: frequência cardíaca, respiração, irritabilidade reflexa, tônus musculares e cor. Para a análise o escore do Apgar foi simplificado em < 7 e em $\geq 7^{3}$

Foram consideradas as variáveis a seguir: os nascidos vivos com idade gestacional entre $20^{a}$ e $37^{a}$ semanas, idade, raça-cor, profissão, estado 
civil, além do tipo de parto da puérpera. Em relação ao prematuro foi revisada a ficha de admissão neonatal, onde foram verificadas informações referentes ao sexo, peso ao nascer, Apgar e se teve alguma anomalia ou malformação congênita.

Na análise dos dados foram utilizadas medidas de frequências absolutas e relativas. A organização do banco de dados foi no Microsoft Office Excel, versão 2010, e a análise das variáveis nos softwares Minitab v.14 ${ }^{16}$. SPSS v. $20^{17}$ e Epi Info v.618.

O estudo contou com a apreciação do Comitê de Ética em Pesquisa da Universidade do Estado do Amazonas e da Secretária Municipal de Saúde do Município de Coari, sendo aprovado mediante parecer $n^{\circ} 1.787 .260$ de 22.10.2016, que, em se tratando de dados secundários, dispensou o Termo de Consentimento Livre e Esclarecido (TCL). Porém, garantiu o anonimato e confidencialidade das informações nos prontuários.

\section{RESULTADOS}

No período de 2011 a 2015, foram notificadas 957 gestantes que tiveram seus partos prematuros no Centro Obstétrico do Hospital Regional Dr. Odair Carlos Geraldo. O número de partos para cada ano foram: 2011(204), 2012 (196), 2013 (191), 2014(137) e 2015 (229). Dentre os anos pesquisados pode-se observar que 2015 foi o ano que teve relativamente a maior concentração de mulheres com partos prematuros (23,9\%) (Figura 1).

Figura 1 Distribuição dos partos prematuros no Centro Obstétrico de Coari, Amazonas, no período de 2011 a 2015.

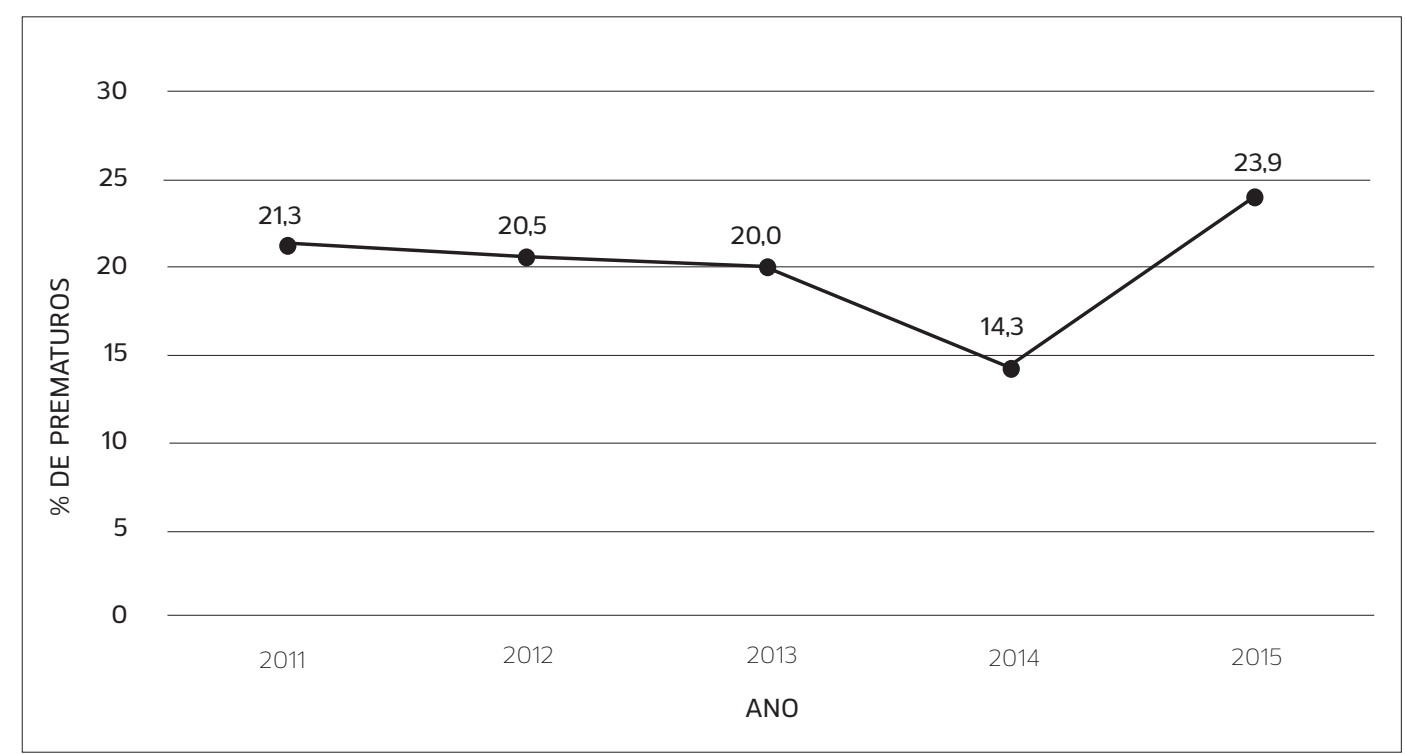

Fonte: Elaborado pelos autores (2018)

Os dados sociodemográficos das mães mostraram que a maioria estava na faixa etária entre 15 e 19 anos, num total de 337 (35,3\%), e evidenciou-se uma parcela importante de crianças grávidas na faixa etária de 10 a 14 anos - 45 (4,7\%) (Tabela 1).

No referente à variável raça/cor observou-se que 892 (95,8\%) das mães se autodeclaravam como pardas. A análise da variável ocupação mostrou que 300 (32,4\%) eram agricultoras, seguidas das donas do lar 265 (28,6\%) e estudantes que apresentaram um percentual próximo 240 (25,9\%), já para o estado civil dominaram as outras 532 (56,8\%), totalizando 532 (Tabela 1). 
Tabela 1. Características sociodemográficas das mães dos prematuros nascidos no município de Coari, Amazonas, no período de janeiro de 2011 a dezembro de 2015.

\begin{tabular}{|c|c|c|}
\hline Variáveis & $\mathbf{n}$ & $\%$ \\
\hline \multicolumn{3}{|l|}{ Faixa Etária } \\
\hline $10-14$ & 45 & 4,7 \\
\hline $15-19$ & 337 & 35,3 \\
\hline $20-24$ & 245 & 25,7 \\
\hline $25-29$ & 190 & 19,9 \\
\hline $30-34$ & 88 & 9,2 \\
\hline $35-39$ & 36 & 3,8 \\
\hline $40-44$ & 13 & 1,4 \\
\hline $45-49$ & 1 & 0,1 \\
\hline Ignorado/em branco/ilegíveis* & 2 & \\
\hline \multicolumn{3}{|l|}{ Raça/cor } \\
\hline Parda & 892 & 95,8 \\
\hline Branca & 36 & 3,9 \\
\hline Preta & 3 & $>0,003$ \\
\hline Ignorado/em branco/ilegíveis* & 26 & \\
\hline \multicolumn{3}{|l|}{ Profissão } \\
\hline Agricultora & 300 & 32,4 \\
\hline Assalariada & 107 & 11,5 \\
\hline Do lar & 265 & 28,6 \\
\hline Estudante & 240 & 25,9 \\
\hline Autônoma & 15 & 1,6 \\
\hline Ignorado/em branco/ilegíveis* & 30 & \\
\hline \multicolumn{3}{|l|}{ Estado Civil } \\
\hline Casada & 96 & 10,2 \\
\hline Solteira & 532 & 56,8 \\
\hline União Estável & 309 & 33,0 \\
\hline Ignorado/em branco/ilegíveis* & 20 & \\
\hline
\end{tabular}

A tabela 2 apresenta a situação obstétrica das gestantes e condições do parto prematuro. A avaliação da idade gestacional mostrou que o maior percentual estava entre 27 e 47 semanas e mais 924 (96,7\%), e entre 14 e 26 semanas foi de 32 (3,3\%). Quanto ao tipo de parto foi observado que 620 (65,0\%) foram partos normais e 334 (35,0\%) partos cesarianos. No entanto, dentre as causas mais frequentes que as mulheres referiram para o parto prematuro foi a contração uterina, 759 (79,1\%), seguida de perda de líquido amniótico 77 (8,0\%).

Sobre o sexo dos prematuros, predominou o masculino, 497 (52,7\%), no concernente à prematuridade foram moderados, 481 (50,3\%), e com idade gestacional de 35 a 37 semanas, 701 (73,3\%), conforme mostra a tabela 3 . Referente ao peso ao nascer, 662 (72,3\%) tiveram com peso acima de $2.500 \mathrm{~kg}$ (Tabela 3).

Verificou-se em relação ao índice de Apgar. que 98 (10,6\%) dos prematuros obtiveram pontuação < 7 no primeiro minuto de vida, sendo que no quinto minuto esse percentual caiu para 72 (7,8\%), indicando que a maioria teve Apgar $\geq 7$. Além disso, houve má formação congênita em 29 (3,3\%) dos neonatos (Tabela 3). 
Tabela 2. Situação obstétrica e do parto prematuro das gestantes no período de janeiro de 2011 a dezembro de 2015, no Município de Coari - Amazonas

\begin{tabular}{lcc}
\hline Situação obstétrica & $\mathbf{n}$ & $\%$ \\
\hline Tipos de Parto & & \\
\hline Normal & 620 & 65,0 \\
\hline Cesárea & 334 & 35,0 \\
\hline Ignorado/em branco/ilegíveis* & 3 & \\
\hline Diagnóstico do Parto*** & & 79,1 \\
\hline Contração Uterina & 759 & 1,0 \\
\hline Dor baixo ventre & 10 & 2,3 \\
\hline Óbito fetal & 22 & 1,8 \\
\hline Sangramento Vaginal & 17 & 2,8 \\
\hline Pré - eclampsia & 20 & 8,0 \\
\hline Perda de Líquido Amniótico & 77 & 1,0 \\
\hline Pico hipertensivo & 10 & 4,5 \\
\hline Outros & 45 & \\
\hline Ignorados/brancos/ilegíveis* & 10 & \\
\hline
\end{tabular}

Fonte: Elaborado pelos autores (2018)

*Os casos ignorado/em branco/ilegíveis são apresentados como informativos, mas excluídos das análises.

**Nota: o número de eventos é maior aos pacientes por causa de algumas parturientes apresentarem múltiplos sintomas na hora do parto.

***Outros: junção de alguns eventos que de maneira isolada seriam pouco expressivos (por exemplo, sofrimento fetal e desproporção céfalo-pélvica).

Tabela 3. Características das crianças prematuras que nasceram no Centro Obstétrico de Coari, Amazonas, no período de janeiro de 2011 a dezembro de 2015.

\begin{tabular}{|c|c|c|}
\hline Variáveis & $\mathbf{n}$ & $\%$ \\
\hline \multicolumn{3}{|l|}{ Sexo do prematuro } \\
\hline Masculino & 497 & 52,7 \\
\hline Feminino & 444 & 47,1 \\
\hline Indefinido & 2 & 0,2 \\
\hline Ignorado/em branco/ilegíveis* & 14 & \\
\hline \multicolumn{3}{|l|}{ Peso do prematuro } \\
\hline$\leq 2.500 \mathrm{~g}$ & 253 & 27,7 \\
\hline$>2.500 \mathrm{~g}$ & 662 & 72,3 \\
\hline Ignorado/em branco/ilegíveis* & 42 & \\
\hline \multicolumn{3}{|l|}{ Tipo de prematuro } \\
\hline Extremo & 59 & 6,2 \\
\hline Limítrofe & 401 & 41,9 \\
\hline Moderado & 481 & 50,3 \\
\hline Supra extremo & 15 & 1,6 \\
\hline Ignorado/em branco/ilegíveis* & 1 & \\
\hline \multicolumn{3}{|l|}{ Idade gestacional (semanas) } \\
\hline $20-24$ & 21 & 2,2 \\
\hline $25-29$ & 45 & 4,7 \\
\hline $30-34$ & 189 & 19,8 \\
\hline $35-37$ & 701 & 73,3 \\
\hline Ignorado/em branco/ilegíveis* & 1 & \\
\hline
\end{tabular}




\begin{tabular}{lcc}
\hline Variáveis & $\mathbf{n}$ & \% \\
\hline Apgar no primeiro minuto & & \\
\hline$<7$ & 88 & 10,6 \\
\hline$\geq 7$ & 29 & 89,4 \\
\hline Ignorado/em branco/ilegíveis ${ }^{* *}$ & & \\
\hline Apgar no quinto minuto & 72 & 7,8 \\
\hline$<7$ & 856 & 92,2 \\
\hline$\geq 7$ & 29 & \\
\hline Ignorado/em branco/ilegíveis* & & 36,7 \\
\hline Anomalias congênitas & 838 & 3,3 \\
\hline Não & 29 & \\
\hline Sim & 90 & \\
\hline Ignorado/em branco/ilegíveis* & & \\
\hline
\end{tabular}

Fonte: Elaborado pelos autores (2018)

*Os casos ignorado/em branco/ilegíveis são apresentados como informativos, mas excluídos das análises.

\section{DISCUSSÃo}

Foram levantados 957 casos de crianças prematuras nascidas no Centro Obstétrico do Hospital Regional Dr. Odair Carlos Geraldo, no período de janeiro de 2011 a dezembro de 2015. Os percentuais dos casos de partos prematuros notificados permanecem bem próximos durante todos os anos, com exceção para o ano de 2014, o que pode ter sido ocasionado pela crise política e de gestão que atravessou o município, principalmente caracterizado por apresentar uma alta rotatividade de gestores nas diversas secretarias.

As características sociodemográficas mostraram que a faixa etária que se destacou foi de 15 a 19 anos com 35,3\%, e a faixa etária de 20 a 24 anos com 25,7\%, sendo semelhante a outros estudos realizados no Brasil, Região Norte, e Amazonas ${ }^{19}$, onde um dos fatores de risco predominante na interrupção da gravidez é a idade materna, especialmente, na adolescência em Coari. Estudo sobre prematuridade realizado no Brasil em $2016^{19}$, revelou 55,5\% de adolescentes grávidas para o Brasil, 52,4\% para a região Sudeste, e o maior percentual de 67,4\% correspondeu à região Norte.

Pesquisa realizada na reserva de Mamirauá, interior do Amazonas, mostrou gravidez em 81,0\% das adolescentes na faixa etária de 10 a 19 anos $^{20}$. Fato corroborado por estudo realizado em 2010²1. o qual afirma que, no Brasil, as adolescentes jovens apresentaram 75,0\% de chance de ter partos prematuros, quando comparadas com mulheres adultas. Essas complicações que ocorrem durante o período gravídico em adolescentes podem trazer mais consequências negativas para os neonatos do que para a genitora, podendo-se citar o baixo peso, o índice de Apgar insatisfatório e a morbimortalidade desses recém-nascidos ${ }^{22}$. Porém, vale ressaltar que a prematuridade está mais relacionada com as condições socioeconômicas da gestante do que com a idade materna, o baixo número de consultas do pré-natal ou da ausência delas, bem como de um acompanhamento inadequado ${ }^{23.24}$.

Quanto à categoria raça/cor um percentual significativo se autodeclarou como pardas (95,8\%). Corroborando os resultados obtidos em pesquisas realizadas em 2011 e 2012 25,26, que encontraram um maior percentual de mães com partos prematuros, que se autodeclararam pardas, ressaltando que este fato pode encontrar explicação na miscigenação existente no Brasil. Porém, um estudo realizado em $2015^{27}$ encontrou resultados contrários, onde predominaram mulheres negras, com situação 
financeira desfavorável e dificuldades de acesso aos serviços de saúde, quando comparadas com mulheres pardas e brancas. Porém, o estudo anterior concorda com outro realizado no Brasil em 2016'19, onde as mulheres brancas apresentaram menor probabilidade de uma gravidez precoce, enquanto que esta aumentava quando se tratava de mulheres pretas e indígenas. De maneira que as altas taxas de gravidez na adolescência mostram relação com fatores socioeconômicos e com minorias raciais e étnicas, quando comparadas com mulheres brancas ${ }^{28}$.

Houve uma predominância neste estudo de mulheres que trabalhavam como agricultoras (32,4\%), seguidas das donas do lar (28,6\%). Uma pesquisa realizada em Campo Grande em $2011^{29}$ mostrou que a maioria das mães de prematuros era dona do lar, discordando dos nossos achados, mas eles consideraram esse fator importante para o risco de uma gestação interrompida. Assim também, uma pesquisa no Rio Grande do Norte evidenciou que os maiores índices de partos prematuros aconteciam com donas de $\operatorname{casa}^{30}$. No entanto, outros estudos mostraram que mulheres com precárias condições de saúde e socioeconômicas são mais suscetíveis ao parto prematuro do que as mulheres de classe média e alta ${ }^{29,31}$.

A situação conjugal é um fator que chama a atenção para o parto prematuro, e esta pesquisa mostra que 56,8\% das puérperas eram mães solteiras. Um estudo na cidade de Manaus constatou que as mães solteiras representaram 53,0\% dos casos de prematuridade ${ }^{32}$. Uma pesquisa realizada constatou que o abandono por parte dos cônjuges é muito significativo para as mães, já que se sentem angustiadas ao pensar que terão que criar uma criança sozinhas ${ }^{33}$. Outros pesquisadores ressaltam ainda que o estado civil pode ser um fator de risco relevante para a prematuridade, já que durante a gestação a mulher fica mais fragilizada e vulnerável em termos emocionais, com preocupações que podem causar estresse, pois precisa de apoio e compartilhar as responsabilidades do bebê29. 34 .

Em relação ao tipo de parto, neste estudo observou-se que $65,0 \%$ das mulheres tiveram partos normais e 35,0\% cesáreos. O parto normal tem benefícios comprovados para a mãe e filho, tais como melhor desenvolvimento da criança, rápida recuperação da mãe, convívio mais cedo com a família e sociedade, pois a dor do parto é um processo natural na vida da maioria das mulheres ${ }^{35}$. É a partir daí que o parto normal precisa tornar-se um ambiente sociável que não seja restrito a locais cirúrgicos, para que seja amenizada a ansiedade, tensão antes do parto e o sofrimento que pode representar em diversas ocasiões a opção pelo parto cesariano ${ }^{35}$

Os dados deste estudo apontam um percentual importante de partos cesáreos. O Brasil ocupou, durante alguns anos, uma posição nada admirável de campeão de cirurgias cesarianas ${ }^{22}$, o que pode ser indicativo da desvalorização do parto normal e da falta de informação, educação em saúde e incapacidade de escolha das mulheres de práticas de intervenção quando confrontadas com as decisões técnicas dos profissionais de saúde ${ }^{22,36}$. Estudo realizado em Caxias do Sul em 2012 identificou que a cesárea mostrava uma associação com o maior risco de mortalidade neonatal de fetos de gravidez de baixo risco, porém pode dar alguma vantagem de sobrevivência para os de menos de 26 semanas $^{28}$.

Segundo o Ministério da Saúde ${ }^{37}$. o parto cesáreo não deveria acontecer, pois a retirada da criança pode causar sérias complicações na sua vida, assim como algumas dificuldades para a mãe, como possíveis infecções e, até mesmo complicações devido à anestesia. Durante o momento do parto são liberadas algumas substâncias e hormônios que auxiliam no desenvolvimento da criança e amamentação ${ }^{38}$.

O parto cesariano é recomendado geralmente em gravidez de risco ou casos excepcionais, sendo que quando o primeiro parto é desse tipo há uma maior probabilidade do segundo também ser como o primeiro ${ }^{26}$, porém, ele não traz quase benefício para a mãe e para o bebê, uma vez que um estudo realizado mostrou que o parto cesariano demanda um tempo maior de recuperação para a mãe, expondo a gestante a processos infecciosos e 
hemorrágicos, além disso, este deve ser feito por profissionais especializados, para diminuição dos riscos tanto para a mãe, quanto para o bebê ${ }^{39}$.

No município de Coari, os principais sinais de parto prematuro foram as contrações uterinas (79,1\%). O trabalho de parto prematuro pode apresentar alguns sinais e sintomas, e dentre eles está a contração uterina, com presença de dor ou sem dor ${ }^{40}$. Estudo realizado em Imperatriz, Maranhão, em $2012^{26}$, mostrou que as complicações mais graves durante a gestação foram as infecções do trato urinário, as doenças hipertensivas e o sangramento vaginal.

Com relação aos dados obtidos na pesquisa sobre prematuros, constatou-se que predominou o sexo masculino (52,7\%). Os bebês de sexo masculino são mais propensos a nascer antes do tempo quando comparados aos do sexo feminino ${ }^{41}$.

Esta pesquisa mostrou que a maioria dos neonatos prematuros apresentava peso dentro dos parâmetros de normalidade de $>2.500 \mathrm{~g}$ (72,3\%), sendo que também se destacaram os nascidos entre 35 e 37 semanas de gestação, 73,3\%. Estudo realizado em Manaus em 2015,42 evidenciou que o maior percentual de crianças prematuras nasceu com o peso adequado, superior a $2.500 \mathrm{~g}$, e $93,5 \%$ dos casos estavam com 37 semanas de gestação. Porém, crianças com o peso $\leq 2.500$ g são os mais suscetíveis a uma hospitalização e desenvolvimento comprometido ${ }^{43}$. Outros estudos apontam que a condição socioeconômica e de saúde da mãe contribuem para o baixo peso da criança, podendo afetar o desenvolvimento normal e favorecer a morbimortalidade neonatal ${ }^{44,45}$.

A análise do índice de Apgar no quinto minuto de vida revela que, entre todos os prematuros, um percentual de 7,8\% obteve um índice insatisfatório no mesmo. Achados de estudo no Rio Grande Sul coincidem com estes resultados, já que houve predominância dos parâmetros normais, diminuindo assim um risco para a saúde do neonato ${ }^{46,47}$. O Apgar inferior a 7 no $5^{\circ}$ minuto é um alerta de risco para o neonato adquirir alguns problemas neurológicos e as chances de sobrevida são menores. O mesmo estudo também mostrou que os neonatos com muito baixo peso sofrem hipóxia no $1^{\circ}$ minuto de vida se seu Apgar de $0-3^{48}$.

No presente estudo também foi identificado um percentual de 3,3\% de crianças que nasceram com algum tipo de malformação congênita. Esta é a segunda maior causa de morbidade e mortalidade no Brasil ${ }^{35}$. A malformação pode interromper a gestação, portanto, para salvaguardar a vida da genitora como a do feto, nesses casos, geralmente, é realizado o parto cesariano ${ }^{29}$.

A gravidez na adolescência vem crescendo no município, constituindo um fator importante no aumento das chances de gestantes terem parto prematuro. No tocante ao neonato prematuro, o Hospital Regional de Coari, no momento da realização da pesquisa, não contava com UTI neonatal e nem com profissionais especializados na assistência ao mesmo, o que pode comprometer as chances de sobrevida dos prematuros. A prematuridade não pode ser evitada, porém, pode ser prevenida, por isso é muito importante a realização do pré-natal, para acompanhar o estado de saúde da mãe e da criança.

Há de se ressaltar que entre os resultados sociodemográficos das mães do presente estudo, observou-se uma parcela importante de crianças grávidas na faixa etária de 10 a 14 anos (4,7\%). A saúde materna é cercada de diversos agravos e complicações perinatais quando a gravidez acontece durante a adolescência, ou pior ainda, quando estamos falando de crianças que nem sequer alcançaram um nível de desenvolvimento do organismo, o que termina predispondo uma situação potencial de risco tanto para a mãe como para o recém-nascido, assim como apresentam uma responsabilidade precoce, despreparo de responsabilidade social, econômico e psicológico 22.49 .

A gestação em adolescente já é considerada um problema de saúde pública e não é difícil imaginar quando esta situação acontece com crianças que engravidam em decorrência de violência sexual, porque toda criança menor de 18 anos grávida, o Estatuto da Criança e do Adolescente (ECA)" considera estupro de vulnerável. O que, além de antecipar alguns processos, termina em sofrimento 
psíquico e impedindo de acontecer o processo maturativo em curso ${ }^{50}$.

Assim, a mulher grávida em decorrência de violência sexual tem o direito de ser informada sobre as alternativas legais com relação ao destino da gestação, prevenção de doenças e possibilidades de atenção à saúde ${ }^{50}$. Porém, uma pesquisa sobre o estupro no Brasil em $2014^{51}$ constatou que quanto menor for a escolaridade, as crianças/adolescentes recebiam um tratamento diferenciado no que diz respeito ao princípio da universalidade e agravado mais ainda em se tratando de residentes em área rurais, refletindo diretamente na qualidade dos serviços públicos ofertados ${ }^{51}$.

\section{CONCLUSÕES}

O presente artigo traz pela primeira vez a discussão do nascimento de prematuros no município de Coari, mostrando uma importante constatação de crianças grávidas, possivelmente vítimas de violência sexual, o que termina gerando custos para o Sistema Único de Saúde e para a vitima, que seja desde a perspectiva de tratamento de traumas psicológicos e sociais dessas crianças grávidas, já que as mesmas estão despreparadas para assumir responsabilidades de cuidado de outra criança, ou desde o ponto de vista de seus filhos prematuros em risco de morte ou de levar uma vida com sequelas, em decorrência de complicações do parto.

O anterior problemática exige incentivar ações de prevenção de gravidez na adolescência, especialmente nas áreas rurais, como uma forma de evitar o aumento de partos prematuros no município. Assim como orientar as gestantes sobre a importância da qualidade do pré-natal e acompanhamento nutricional durante a gestação, buscando sempre a saúde da mãe e do filho.

Proporcionar treinamentos para o bom preenchimento das fichas das gestantes, e assim evitar a falta de informações nos prontuários e dificuldade de leitura;
Esforços devem partir da prefeitura e de gestores em saúde para realizar a construção de uma maternidade com boa infraestrutura física e de recursos humanos especialistas na área neonatal, justificada pelo elevado número de partos prematuros e prevenção de mortes neonatais;

O desenvolvimento social e a garantia de direitos básicos de dignidade e respeito à sexualidade das crianças coarienses exigem a superação de uma cultura machista e o quadro de violações sexuais históricas, que impõem diversos desafios, em termos de políticas públicas e de gestão municipal que visem a garantia do respeito aos Direitos Humanos de crianças e adolescentes.

\section{REFERÊNCIAS}

1. Rocha RCL, Souza E, Guazzelli CAF, Filho AC, Soares EP, Nogueira ES. Prematuridade e baixo peso entre recém-nascidos de adolescentes primíparas. Rev Bras Ginecol Obstet [Internet]. 2006 Mar [citado 2015 Mar 05]:28(9):530-5. Disponível em: http://www.scielo.br/pdf/rbgo/v28n9/05.pdf.

2. Bittar RE, Zugaib M. Indicadores de risco para o parto prematuro. Rev Bras Ginecol Obstet. [Internet]. 2009 Out [citado 2017 Out 15];31(4):203-9. Disponivel em: http://www.scielo.br/pdf/ rbgo/v31n4/08.pdf.

3. Santos LM dos, Pasquini VZ. A importância do Índice de Apgar. Rev Enferm. UNISA [Internet]. 2009 Ago [citado 2016 Ago 13]:10(1):39-43. Disponivel em: http://www.unisa.br/graduacao/ biologicas/enfer/revista/arquivos/2009-1-08.pdf.

4. Rades E, Bittar RE, Zugaib M. Determinantes diretos do parto prematuro eletivo e os resultados neonatais. Rev Bras Ginecol. Obstet. [Internet]. 2004 Jul [citado 2018 Jul 25]; 26(8):6655-62. Disponivel em: http://www.scielo.br/scielo.php?script=sci_artte xt\&pid=S010072032004000800010\&lng=en.

5. Souza LF, Botelho NM. Fatores de risco para o parto prematuro em puérperas que tiveram parto pré-termo. Rev para. Med. [Internet]. 2011 Ago [citado 2016 Ago 1];24(e2750):2-8. Disponível em: http://files.bvs.br/upload/S/0101-5907/2011/v25n4/a3056. pdf.

6. Rocha RCL, Souza E de, Guazzelli CAF. Filho AC, Soares EP, Nogueira ES. Prematuridade e baixo peso entre recém-nascidos de adolescentes primíparas. Rev Bras Ginecol Obstet. [Internet]. 2006 Abr [citado 2018 Abr 19]:28(9):530-5. Disponivel em: http://www.scielo.br/pdf/rbgo/v28n9/05.pdf.

7. Andrade PC., Linhares JJ, Martinelli S, Antonini M, Lippi UG, Baracat F. Resultados perinatais em grávidas com mais de 35 Anos:estudo controlado. RBGO 2004 Set [citado 2015 Set 05]:26 (9):697-702. Disponivel em: http://dx.doi.org/10.1590/S010072032004000900004 .

8. Goldenberg P. Figueiredo MCT, Silva RS. Gravidez na adolescência, pré-natal e resultados perinatais em Montes Claros, 
Minas Gerais, Brasil. Cad. Saúde Pública. 2005 Set [citado 2015 Set 05]:21(4):1077-86. Disponivel em: http://dx.doi.org/10.1590/ S0102-311X2005000400010.

9. Centers for Disease Control and Prevention. Department of Health and Human Services. CDC Health Disparities and Inequalities Report- United States, 2013. Morbidity and Mortality Weekly Report [citado 10 janeiro 2016]: 62(3). Disponível em: www.cdc.gov/mmwr/pdf/other/su6203.pdf.

10. Cohen WR. Does maternal age affect pregnancy outcome? Royal College of Obstetricians and Gynecologists'. BJOG [Internet]. 2014 Jan [citado 2016 Jan 10]:121:252-4. Disponivel em: http://onlinelibrary.wiley.com/doi/10.1111/1471-0528.12563/pdf.

11. Novaes CEF, Koch HA, Montenegro CAB, Rezende Filho, JF de. Diagnóstico do parto pré-termo pela medida ultrassonográfica do comprimento do colo uterino. Radiol Bras [Internet]. 2009 Maio [citado 2018 Maio 09]:42 (5):295-8. Disponivel em: http://www. scielo.br/pdf/rb/v42n5/a07v42n5.pdf.

12. Organização Mundial da Saúde. Born too soon: the global action report on preterm birth [Internet]. Geneva: OMS; 2012 [citado 2016 Fev 20]. Disponivel em: http://whqlibdoc.who.int/ publications/2012/9789241503433_eng.pdf.

13. Barizon TO, Santos JR, Reis CB. Fatores relacionados à prematuridade dos recém-nascidos de Dourados nos anos de 2011 e 2012. [Tese]. Cáceres (MG): Universidade Estadual de Mato Grosso; 2014 Agost [citado 2018 Agost 20]. Disponivel em: https://anaisonline.uems.br/index.php/enic/article/view/2396.

14. Duarte MMP, Freire EEG, Oliveira JFB de. Assistência de enfermagem à gestante em trabalho de parto prematuro. Interface: Comunic., Saude, Educ.. 2015 Mar [citado 2016 Mar 16]:3 (1):1-8. Disponivel em: http://dx.doi.org/10.16891/ 2317.434X.143.

15. Figueiró-Filho EA, Oliveira VM de, Ferreira CM, Silva VM da, Silva Tinos AL da, Kanomata LB. Variáveis perinatais e associação de recém-nascidos de muito baixo peso ao nascer em hospital público universitário do Brasil. Rev Bras Ginecol Obstet. [Internet 2014 Set [citado 2016 Set 5]: 36(1):10-6. Disponivel em: http:// www.scielo.br/pdf/rbgo/v36n1/0100-7203-rbgo-36-01-00010. pdf.

16. Minitab 14 Statistical Software Academic, State College, PA: Minitab; 2003.

17. IBM Corp. Released 2011. IBM SPSS Statistics for Windows, Version 20.0. Armonk, NY: IBM Corp.

18. WHO (World Health Organization), 1994. Epi Info. Version. 6.02. A Word Processing. Database, and Statistics System for Epidemiology on Microcomputers. Geneva: WHO.

19. Cruz MS da, Carvalho FJV, Irffi G. Perfil socioeconômico, demográfico, cultural, regional e comportamental da gravidez na adolescência no Brasil. Plan Pol Públ.(PPP) [Internet]. 2016 Jul [citado 2018 Jul 7]:46:243-66. Disponivel em: http:// repositorio.ipea.gov.br/bitstream/11058/6577/1/ppp_n46_perfil_ socioeconomico.pdf.

20. Ruzany MH, Tavares Andrade CL de, Meirelles ZV, Moura EAF, Deusdará R, Rodrigues V. et al. Desinformação e vulnerabilidades com relação à sexualidade dos adolescentes e jovens da Reserva de Mamirauá, Amazonas-Brasil. Adolesc. Saude [Internet] 2010 Out [citado 20160ut 6]; 7(2): 41-49. Disponível em: http:// adolescenciaesaude.com/detalhe_artigo.asp?id=188\#.

21. Martins EF. Mortalidade perinatal e avaliação da assistência ao pré-natal, ao parto e ao recém-nascido em Belo Horizonte. [Tese]: Minas Gerais. Universidade Federal de Minas Gerais; 2010. Disponivel em: http://www.bibliotecadigital.ufmg.br/.
22. Ramos HAC, Cuman RKN. Fatores de risco para prematuridade: pesquisa documental. Esc Anna Nery Rev Enferm. [Internet] 2009 Abr [citado 2016 Abr 4];13(2):297-304. Disponivel: http:// www.redalyc.org/pdf/1277/127715322009.pdf.

23. Oyamada LH, Mafra PC, Meireles R de A, Guerreiro TMG. Caires Júnior MO de, Silva FM da. Gravidez na adolescência e o risco para a gestante. Brazilian Journal of Surgery and Clinical Research - BJSCR [Internet]. 2014 Ago [citado 2018 Agost 21];6(2):38-45. Disponivel em: http://www.mastereditora.com.br/ periodico/20140331_212052.pdf.

24. Azevedo WF de, Diniz MB, Fonseca ESVB da, Azevedo LMR de, Evangelista, CB. Complicações da gravidez na adolescência: revisão sistemática da literatura. Rev Einstein. [Internet] 2015 Jun [citado 2018 Jun 01]:13(4):618-26. Disponivel em: http:// www.scielo.br/pdf/eins/v13n4/pt_1679-4508-eins-S167945082015RW3127.pdf.

25. Lima CRV. Silva CS. Perfil sócio demográfico clínico e obstétrico de gestantes admitidas numa maternidade de referência do Recife com diagnóstico de parto prematuro no ano de 2010. Revista Conceito A [Internet]. 2011 Ago [citado 2018 Agost 19]:2:64682. Disponivel em: http://www.faculdadesaomiguel.com.br/pdf/ revista-conceito/n2/enfermagem/perfil-socio-demografico.pdf.

26. Almeida AC de, Jesus ACP de, Lima PFT, Araújo MFM de, Araújo TM de Fatores de risco maternos para prematuridade em uma maternidade pública de Imperatriz-MA. Rev Gaúcha de Enferm. [Internet]. 2012 Jun [citado 2018 Jun 9];33(2):86-94. Disponivel em: http://www.scielo.br/pdf/rgenf/v33n2/13.pdf.

27. Alves TL, Costa Ribeiro Júnior H da, Lima Costa M de, Valois SS. Fatores associados ao recém-nascido pequeno para a idade gestacional: uma revisão. Nutrire. [Internet] 2015 Set [citado 2017 Set 05]:40(3):376-382. Disponivel em: http://dx.doi. org/10.4322/2316-7874.04513.

28. Madi JM, Araújo BF de, Zatti H, Rombaldi RL, Lorencetti J, Pinson G, et al. Avaliação dos fatores associados à ocorrência de prematuridade em um hospital terciário de ensino. Revista da AMRIGS [Internet]. 2012 Agos [citado 2018 Agos 13];56(2):111-118. Disponivel em: http://www.amrigs.com.br/revista/56-02/original2. pdf.

29. Nogarett DM. Anache AA. Análise dos determinantes do nascimento de crianças pré-termo segundo os registros dos prontuários médicos das parturientes internadas no Hospital Universitário Maria Aparecida Pedrossian - Cg- Ms. In:VII Encontro da Associação Brasileira de Pesquisadores em Educação Especial [Internet]; 2011 Nov 08-10; Londrina. Londrina: UEL, 2011. [citado em 2018 Out 22]. Disponivel em: http://www.uel. br/eventos/congressomultidisciplinar/pages/arquivos/anais/2011/ AVALIACAO/155-2011.pdf.

30. Holanda RMO. Prevalência e fatores associados à asfixia perinatal a partir de uma amostra de nascidos vivos [dissertação]. Mossoró: Universidade do Estado de Rio Grande do Norte; 2015.

31. Freitas PF, Araújo RR de. Prematuridade e fatores associados em Santa Catarina, Brasil: análise após alteração do campo idade gestacional na Declaração de Nascidos Vivos. Rev Bras Saúde Mater. Infant. [Internet] 2015 Nov [citado 2017 Nov 16]:15(3). Disponivel em: http://www.scielo.br/pdf/rbsmi/v15n3/1519-3829rbsmi-15-03-0309.pdf.

32. Cruz MS da. Perfil socioeconômico, demográfico, cultural, regional e comportamental da gravidez na adolescência no Brasil [tese] Paraiba (PE): Universidade Federal da Paraíba; 2016. http:// repositorio.ipea.gov.br/bitstream/11058/6577/1/ppp_n46_perfil_ socioeconomico.pdf" 
33. Balbino SC. Características das mães adolescentes e de seus filhos prematuros no município de Iturama/MG. [Internet] [monografia]. Uberaba (MG): Universidade Federal de Minas Gerais; 2013. [acesso 2018 Out 22]Disponivel em: https://www.nescon. medicina.ufmg.br/biblioteca/registro/referencia/0000003818.

34. Rodrigues APRA, Santos LBB dos, Castela MPS, França AMB de, Pimpão FD. Análise da prematuridade nos extremos da idade reprodutiva. Interf. Cient.-saúde e amb. 2015 Abr [citado 2017 Abr 10];3(3):65-78. Disponível em: https://periodicos.set.edu.br/ index.php/saude/article/viewFile/2107/pdf_4.

35. Lansky S, Lima FAA de, Silva AAM da, Campos D, Azevedo Bittencourt SD de, Carvalho ML de, Frias PG de, Cavalcante RS, Cunha AJLA da. Pesquisa Nascer no Brasil: perfil da mortalidade neonatal e avaliação da assistência à gestante e ao recémnascido. Cad Saúde Pública [Internet]. 2014 Sep [citado 2017 Sep 6]:30(supl.1):S192-S207.Disponivel em: http://www.scielo.br/pdf/ csp/v30s1/0102-311X-csp-30-s1-0192.pdf.

36. Marque FC, Dias IMV, Azevedo L. A percepção da equipe de enfermagem sobre humanização do parto e nascimento. Esc. Anna Nery [Internet]. 2006 [citado 2017 Dez 2]:10(3):439-447. Disponivel: http://eean.edu.br/detalhe_artigo.asp?id=135.

37. Ministério da Saúde, Fundação Oswaldo Cruz. Nascer no Brasil: inquérito nacional sobre parto e nascimento. Cad Saúde Pública [Internet]. 2014 Nov [citado 2017 Nov 29];30(supl. 1):S5. Disponivel: http://www.scielo.br/scielo.php?script=sci_arttext\&pid =S0102311X2014001300001.

38. Prior E, Santhakumaran S, Gale C, Philipps LH, Modi N, J Hyde M. Breastfeeding after cesarean delivery: a systematic review and meta-analysis of world literature. Am J Clin Nutr [Internet]. 2012 Abr [citado 2017 Abr 6]:95(5):1113-1135. Disponivel em: https:// doi.org/10.3945/ajen.111.030254.

39. Padilha JF, Torres RPP, Gasparetto A, Farinha LB, Mattos KM de. Parto e idade: características maternas do estado do Rio Grande do Sul. Saúde (Santa Maria) [Internet]. 2013 Ago [citado 2017 Ago 09];39(2):99-108. Disponivel em: https://periodicos. ufsm.br/revistasaude/article/view/6304/pdf_1

40. Andrade SW. Intercorrências gestacionais: trabalho de parto prematuro [monografia]. Santa Maria: Universidade Franciscana. UNIFRA; 2012

41. Melo WA de; Castellini FM; Alvarenga A, Carvalho, MD. Fatores de riscos obstétricos e neonatais para ocorrência de prematuridade no município de Maringá, PR. In: VII EPCC Encontro Internacional de Produção Científica; 2011 Out 25-28; Maringá, PR. Maringá: Ed. CESUMAR; 2011.

42. Freire JL. Determinantes do baixo peso ao nascer em parturientes atendidas em uma maternidade pública de Manaus, AM-2011 [dissertação]. Manaus: Universidade Federal do Amazonas; 2012

43. Costa EL, Sena MCF, Dias A. Gravidez na adolescência: determinante para prematuridade e baixo peso. Comun Ciênc Saúde [Internet]. 2011 Fev [citado 2018 Fev 14];22(sup. esp. 1): 183-187. Disponivel em: http://repositorio.ipea.gov.br/ bitstream/11058/6577/1/ppp_n46 perfil_socioeconomico.pdf.

44. Vale de Almeida AH do, Costa MCO, Gama SGN da, Amaral MTR, Vieira GO. Baixo peso ao nascer em adolescentes e adultas jovens na Região Nordeste do Brasil. Rev Bras Saúde Mater Infant [Internet] 2014 Set [citado 2017 Set 12];14(3):279-286. Disponivel em: http://www.scielo.br/pdf/rbsmi/v14n3/1519-3829rbsmi-14-03-0279.pdf.

45. Gonzaga ICA, Santos SLD, Silva ARV da, Campelo V. Atenção pré-natal e fatores de risco associados à prematuridade e baixo peso ao nascer em capital do nordeste brasileiro. Ciên Saúde Colet [Internet] 2016 Jan [citado 2018 Jan 16]:21(6):1965-1974. Disponivel em: http://www.scielosp.org/pdf/csc/v21n6/1413-8123csc-21-06-1965.pdf.

46. Bozzetto C, Grave MTQ, Périco E. Incidência de nascimentos prematuros em hospital de um município do Vale do Caí. Destaques Acadêmicos [Internet] 2013 Abr [citado 2018 Abr 4]:5(3). Disponivel em: http://www.univates.br/revistas/index.php/ destaques/article/viewFile/286/282.

47. Renner FW, Garcia EL, Renner JDP, Costa BP, Figueira FP, Ebert JP, et al. Perfil epidemiológico das puérperas e dos recémnascidos atendidos na maternidade de um hospital de referência do interior do Rio Grande do Sul no primeiro semestre de 2014. Boletim Científico de Pediatria [Internet]. 2015 Jun [citado 2018 Jun 19]:4(2):27-32. Disponivel em: http://www.sprs.com.br/ sprs2013/bancoimg/160107101642bcped_v4_n2_a2.pdf.

48. Gaiva MAM, Fujimori E, Sato APS. Mortalidade neonatal em crianças com baixo peso ao nascer. Rev Esc Enferm USP. 2014 Fev [citado 2018 Fev 7]:48(5):778-786.Disponível em: http://www. scielo.br/pdf/reeusp/v48n5/pt_0080-6234-reeusp-48-05-778. pdf.

49. Maciel SSSV, Maciel WV, Lucena Oliveira AG de, Vasconcelos Sobral L de, Vasconcelos Sobral H de, Carvalho ES de, Souza Silva AK de. Epidemiologia da gravidez na adolescência no município de Caruaru, PE. Revista de AMRGS [Internet]. 2012 Mar [citado 2017 Mar 15] 56(1):45-50. Disponível: http://www.amrigs.org.br/ revista/56-1/0000095683-9_954.pdf.

50. Santos SRMLB. Menina, mulher, filha, mãe?: a gravidez decorrente de violência sexual. [tese]. Brasilia: Universidade de Brasília. Instituto de Psicologia; 2013.

51. Cerqueira D, Coelho DSC. Estupro no Brasil: uma radiografia segundo os dados da saúde. Brasília; 2014. (Nota técnica; 11). 